\title{
Three-stage classical molecular dynamics model for simulation of heavy- ion fusion
}

\author{
Subodh S. Godre ${ }^{1, a}$ \\ ${ }^{1}$ Department of Physics, Veer Narmad South Gujarat University, Surat 395007, India
}

\begin{abstract}
A three-stage Classical Molecular Dynamics (3S-CMD) approach for heavy-ion fusion is developed. In this approach the Classical Rigid-Body Dynamics simulation for heavy-ion collision involving light deformed nucleus is initiated on their Rutherford trajectories at very large initial separation. Collision simulation is then followed by relaxation of the rigid-body constrains for one or both the colliding nuclei at distances close to the barrier when the trajectories of all the nucleons are obtained in a Classical Molecular Dynamics approach. This 3S-CMD approach explicitly takes into account not only the long range Coulomb reorientation of the deformed collision partner but also the internal vibrational excitations of one or both the nuclei at distances close to the barrier. The results of the dynamical simulation for ${ }^{24} \mathrm{Mg}+{ }^{208} \mathrm{~Pb}$ collision show significant modification of the fusion barrier and calculated fusion cross sections due to internal excitations.
\end{abstract}

\section{Introduction}

Heavy-ion collisions at near barrier collision energies are dominated by deep-inelastic scattering and fusion which involves large scale transfer of energy from relative motion to internal excitations. These reactions are also strongly affected by the internal structure of the colliding nuclei [1]. For nuclei with static deformation, Coulomb reorientation of the deformed nucleus results in further modification of the barrier parameters [2, 3]. When the two colliding nuclei come very close to the fusion barrier, the shape of the two nuclei may also change due to largescale internal excitations resulting in some modification of the barrier. Therefore, in heavy-ion reaction simulations it is not only necessary to take into account longrange reorientation effect but also the internal excitations of the nuclei at close distances.

In the macroscopic approaches for study of heavy-ion collisions [4, 5] one has to choose relevant collective degrees of freedom describing the overall properties of the nuclei and then either invoke suitable mechanisms for transfer of energy and angular momentum from the collective to the remaining frozen internal degrees of freedom or invoke suitable couplings. Quantum mechanical coupled-channel calculations [6] have been widely used in heavy-ion collision studies close to the barrier. On the other hand, in a completely microscopic approach, which necessarily involves individual particle degrees of freedom, this energy and angular momentum transfer is inbuilt through the solution of the time dependent many body equations of motion and the relevant collective degrees of freedom are determined by the system itself.
Quantum mechanical microscopic approaches like TimeDependent Hartree-Fock (TDHF) [7-10] have been used for studying heavy-ion fusion. The TDHF calculations are, however, very compute intensive.

Since heavy-ions with energies of even a few $\mathrm{MeV} /$ nucleon have short de Broglie wavelength compared to the size of the ions, classical approximations are expected to be good for macroscopic features of heavyion reactions such as fusion. Therefore, various classical macroscopic and microscopic approaches have also been used for study of heavy-ion reaction [2-5, 11-22]. Classical approaches have the advantage of clarity of interpretations and computational ease.

Within the classical approximations, it is possible to include all the degrees of freedom in a completely unconstrained microscopic approach such as Classical Molecular Dynamics (CMD) approach [11-17] or specifically consider only the translational and rotational degrees of freedom of the two nuclei in a Classical RigidBody Dynamics (CRBD) approach to study reorientation effect of a deformed nucleus [2, 3]. Both these approaches individually, however, have their limitations and difficulties.

To overcome the difficulties with the CRBD-model and the CMD-model and at the same time take advantage of both the models, a three-stage Classical Molecular Dynamics (3S-CMD) approach [20] is developed comprising of (i) Rutherford trajectory stage, (ii) CRBD stage and (iii) CMD stage. This model explicitly takes into account not only the long-range reorientation effect at large separations but internal excitations at close separations as well, seamlessly in the same simulation code.

\footnotetext{
${ }^{\mathrm{a}}$ Corresponding author: ssgodre@yahoo.com
} 
The details of the model calculation are given in section 2 and the results of the dynamical simulation and fusion cross section calculations of a light-deformed and heavyspherical system ${ }^{24} \mathrm{Mg}+{ }^{208} \mathrm{~Pb}$ are given section 3. Finally conclusions are given in section 4 .

\section{Calculation details}

\subsection{Nuclei in classical approximation}

Nucleons assumed to be classical point particles without spin interact via suitable two-body forces. The Coulomb potential between protons has the usual form

$$
V_{c}\left(r_{i j}\right)=\frac{1.44}{r_{i j}} \mathrm{MeV}
$$

and the NN potential chosen is a soft-core Gaussian potential given by $[16,23]$ :

$$
V_{N}\left(r_{i j}\right)=-V_{0}\left(1-\frac{C}{r_{i j}}\right) \exp \left(-\frac{r_{i j}^{2}}{r_{0}^{2}}\right)
$$

The NN potential between like particles is taken to be $20 \%$ weaker than that between unlike particles [24].

The distribution of nucleons in each nucleus is first obtained by cyclically minimizing the total potential energy of an initially random distribution of nucleons, with respect to small spatial displacements of individual nucleon coordinates using the code STATIC [16]. The zero-point energy in the ground state is explicitly neglected. However, a purely phenomenological effective NN potential (eq.2) is chosen with its parameters adjusted to reproduce binding energy (B.E.), rms radius (R) and quadrupole deformation parameter $\left(\beta_{2}\right)$ for the cluster of particles in the frozen ground-state. Since for $A \geq 5$ one can have many local minima in the binding energy, a nucleus is chosen which has either the maximum binding energy among the large number of such configurations [18] or alternatively a configuration which is close to the experimental ground-state properties is chosen [19].

A parameter set, $V_{0}=710 \mathrm{MeV}, C=1.88 \mathrm{fm}, r_{0}=1.15$ $\mathrm{fm}$ is used in the present calculation which produces the ground-state properties as given in the table-1.

Table-1: Ground-state properties of the generated nuclei.

\begin{tabular}{|c|c|c|c|c|c|c|}
\hline & \multicolumn{3}{|c|}{ Calculated } & \multicolumn{3}{c|}{ Experimental } \\
\cline { 2 - 7 } & $\begin{array}{c}\text { B.E. } \\
(\mathbf{M e V})\end{array}$ & $\begin{array}{c}\mathbf{R} \\
\text { (fm) }\end{array}$ & $\boldsymbol{\beta}_{\mathbf{2}}$ & $\begin{array}{c}\text { B.E. } \\
(\mathbf{M e V})\end{array}$ & $\begin{array}{c}\mathbf{R} \\
(\mathbf{f m})\end{array}$ & $\boldsymbol{\beta}_{\mathbf{2}}$ \\
\hline${ }^{\mathbf{2 4}} \mathbf{M g}$ & 167.37 & 2.774 & 0.38 & 198.26 & 3.000 & 0.37 \\
\hline${ }^{\mathbf{2 0 8}} \mathbf{P b}$ & 1619.53 & 5.536 & 0.06 & 1636.46 & 5.500 & 0.00 \\
\hline
\end{tabular}

\subsection{Fusion cross sections}

Barrier parameters for $b=0$ (head-on collision) or even at $\mathrm{b}=\mathrm{b}_{c r}$ for a given collision energy and a given initial orientation of the two nuclei are obtained from dynamically generated ion-ion potential. With these parameters, fusion cross section is calculated using the Wong formula [25],

$$
\sigma_{f u s}=\left[\frac{\hbar \omega_{B}}{2 E_{c m}}\right] R_{B}^{2} \ln \left[1+\exp \left(2 \pi \frac{E_{c m}-V_{B}}{\hbar \omega_{B}}\right)\right]
$$

For a given $\mathrm{E}_{\mathrm{cm}}$ a number of random initial orientations for $b=0$ are considered in the present calculation and orientation-averaged fusion cross section is calculated.

\subsection{Classical Molecular Dynamics (CMD-Model)}

All the degree of freedom are included in a completely un-constrained microscopic calculation where trajectories of all the nucleons in both the colliding nuclei are obtained by solving classical coupled equations of motion:

$$
m \frac{d^{2} \boldsymbol{r}_{i}}{d t^{2}}=-\nabla_{i}\left[\sum_{j \neq i} V_{i j}\right]
$$

This model has been used for study of heavy-ion collisions at higher collision energies [11-14] and for low energy collisions [15-17] also. General features of fusion cross sections above the barrier energies were reproduced and it was shown that better agreement with cross sections could be obtained by suitable choice of the potential parameters [16]. These calculations accounted for the size of the nuclei but did not consider the deformation of the constructed nuclei and were initiated at a very small initial separation of $20 \mathrm{fm}$, like in most TDHF calculations.

\subsection{Classical Rigid-Body Dynamics (CRBD- Model)}

This model was developed specifically to study the Coulomb reorientation of a deformed nucleus in heavy-ion collisions $[2,3]$. The model calculation is started at initial separation $\mathrm{R}_{\mathrm{in}}(\mathrm{t}=0)$ where the two nuclei with their ground-state configurations with a given relative orientation between them are placed along their Rutherford trajectories for given collision energy and impact parameter. However, a large initial separation $R_{i n}=2500 \mathrm{fm}$ is required for ${ }^{24} \mathrm{Mg}+{ }^{208} \mathrm{~Pb}$ collision to take into account for the full effect of the torque produced by the long range Coulomb force [3]. The motion of the centre of mass and the orientation of the principal axes of the two colliding nuclei are obtained from numerical solution of the classical equations of motion for rigid bodies [3].

Using the CRBD-model it is shown [3] that the extent of reorientation and the values of the barrier parameters not only depend on the initial orientation but also on the collision energy, contrary to as assumed in most TDHF calculations. The reorientation effect is small at higher energies and becomes significant at collision energies close to the average barrier height in case of collisions involving light deformed nuclei. The CRBD calculations show the importance of correctly incorporating the longrange Coulomb torque on the deformed collision partner in collisions at near-barrier energies; thus emphasizing the importance of obtaining barrier parameters at every value of $E_{\mathrm{cm}}$ for which cross-sections are calculated.

\subsection{Three-Stage Classical Molecular Dynamics (3S-CMD Model)}

Due to the rigid-body constraints in CRBD-model, the two nuclei can not overlap and the energy transfer from the relative motion to internal excitation is neglected even 
after crossing the barrier. No bound state is formed between the two nuclei. Passing over the generated fusion barrier is assumed to be resulting in fusion. It is, however, desirable to take into account the internal excitations and the subsequent capture of the two nuclei behind the fusion barrier. This in-effect can modify the barrier parameters and the calculated fusion cross sections.

The above mentioned problem can be overcome, in principle, by the CMD model $[16,17]$ in which all the degree of freedom are included and the energy transfer between relative and intrinsic motion is in-built. Dissipation of energy can lead to trapping behind the barrier and result in a bound system of the two nuclei. But as noted earlier, the long range reorientation effect requires initiation of the CMD at large distances $\left(R_{\text {in }}=2500 \mathrm{fm}\right)$ [17] as in the CRBD calculations. However, this would result in large computational time as well as accumulation of numerical errors resulting from solving of equations of motions over a large number of the required time steps.

To overcome the above difficulties and at the same time take advantage of the CRBD and the CMD model calculations, a three-stage Classical Molecular Dynamics (3S-CMD) model is developed for heavy-ion collision simulation which combines both the CRBD and CMD model calculations in a common simulation code consecutively [20]. This model explicitly takes into account the long-rage reorientation effect as well.

The 3S-CMD model calculation proceeds in the following three stages:

(1) Rutherford trajectory calculation: The two nuclei, assumed to be charged point particles, are brought along their Rutherford trajectories with given collision energy and impact parameter up to $\mathrm{R}_{\mathrm{in}}=2500 \mathrm{fm}$.

(2) CRBD model calculation: The two nuclei (assumed to be rigid-bodies with their ground-state configuration of nucleon positions) are then allowed to evolve further using the CRBD-model calculation which is continued up to a relatively small separation of $\mathrm{R}_{\mathrm{cm}}=50 \mathrm{fm}$.

(3) CMD calculation: The rigid-body constraints are relaxed at about $\mathrm{R}_{\mathrm{cm}}=50 \mathrm{fm}$ and the trajectories of all the participating nucleons are computed using equations of motion (eq. 4) for all the particles in a CMD-model approach. If required one of the nucleus can still be constrained to be rigid in this stage.

\section{Results and discussions}

Simulation of ${ }^{24} \mathrm{Mg}+{ }^{208} \mathrm{~Pb}$ collision is carried out in the CRBD and 3S-CMD models for $\mathrm{E}_{\mathrm{cm}}=120 \mathrm{MeV}$ and $\mathrm{b}=0$ for an arbitrary initial orientation of the two nuclei. The rotational energy of a nucleus at any instant in the CMD stage of the 3S-CMD simulation is obtained by assuming the nucleus to be a rigid body on the CMD path. The total rotational energy $\left(T_{\text {rot }}\right)$, total internal vibrational excitation energy $\left(\mathrm{T}_{\mathrm{Vib}}\right)$, and total energy of the centre of masses $\left(\mathrm{T}_{\mathrm{cm}}\right)$ at distances close to the barrier are shown in figure-1. This figure also shows $T_{\text {rot }}$ and $T_{c m}$ calculated in the CRBD model which matches well with 3S-CMD calculation, with $\mathrm{T}_{\mathrm{Vib}}=0$, up to distances very close to the barrier top; thus justifying the use of CRBD model as the second-stage in the 3S-CMD model. After crossing the barrier top, $\mathrm{T}_{\mathrm{Vib}}$ rapidly increases, draining the energy from the center of mass motion $\left(\mathrm{T}_{\mathrm{cm}}\right)$ as well as rotational excitation $\left(\mathrm{T}_{\text {rot }}\right)$ and thus resulting in a bound system.

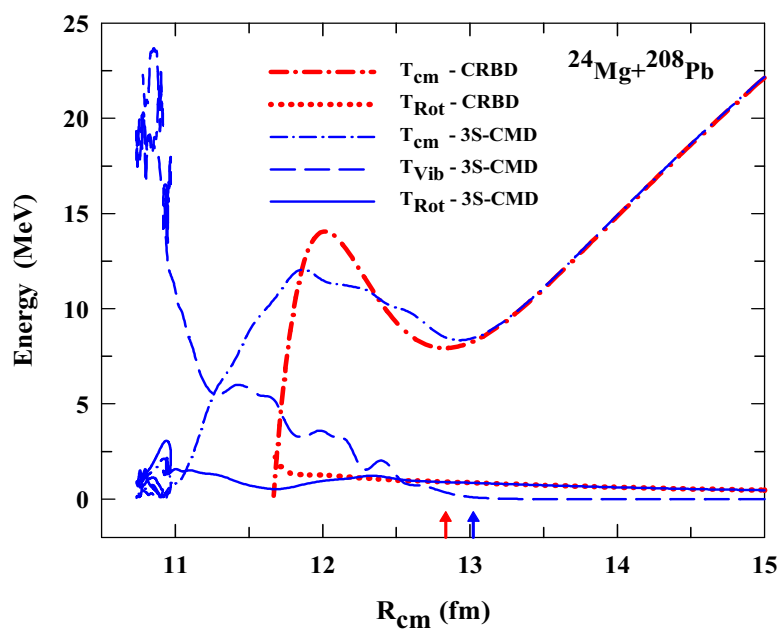

Figure 1: Calculated $\mathrm{T}_{\mathrm{cm}}, \mathrm{T}_{\mathrm{Vib}}$ and $\mathrm{T}_{\mathrm{rot}}$ of ${ }^{24} \mathrm{Mg}+{ }^{208} \mathrm{~Pb}$ collision for CRBD and 3S-CMD [20]. Arrows indicate location of the corresponding barrier top in figure-2.

Figure-2 shows the ion-ion potential calculated in CRBD and 3S-CMD models. Inside of the barrier is significantly modified by the CMD stage of the 3S-CMD. This figure also shows the result of the usual CMD model calculation, initiated only at $R_{c m}=50 \mathrm{fm}$, which does not match well with either CRBD or 3S-CMD calculation.

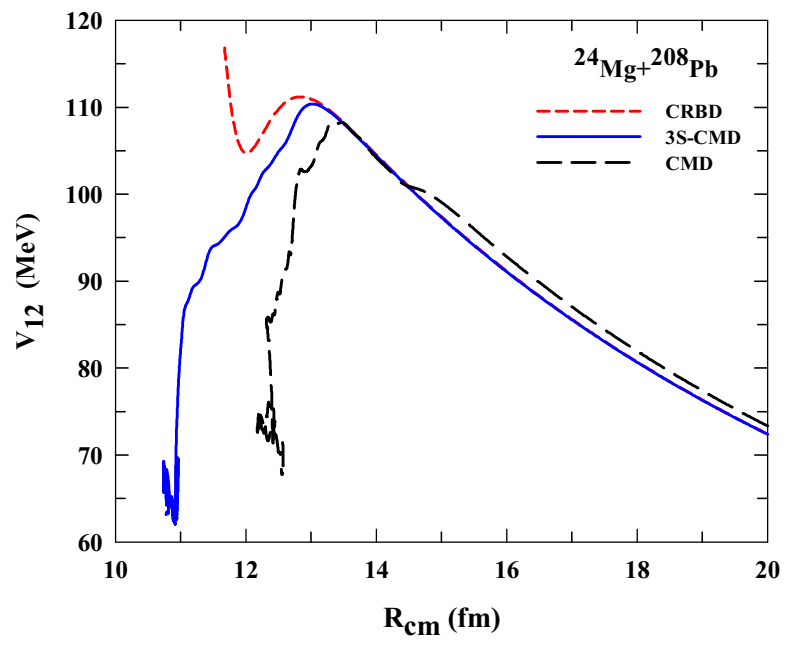

Figure 2: Ion-ion potential for ${ }^{24} \mathrm{Mg}+{ }^{208} \mathrm{~Pb}$ collision in CRBD, CMD and 3S-CMD model simulations [20].

Fusion Cross sections for ${ }^{24} \mathrm{Mg}+{ }^{208} \mathrm{~Pb}$ reaction using CRBD and 3S-CMD models are shown in figure-3 for various assumptions of rigid-body constraint or relaxation of it for one or both the nuclei in the stage- 3 of dynamical simulation. Calculated fusion cross sections keeping ${ }^{24} \mathrm{Mg}$ rigid $(\mathrm{R})$ and ${ }^{208} \mathrm{~Pb}$ non-rigid (NR) (vibrational) in the stage-3, i.e., close to and inside of the barrier, are enhanced at higher energies as compared to the calculations in which ${ }^{208} \mathrm{~Pb}$ is also kept rigid only (CRBD). This enhancement in fusion cross sections arises because of opening up of internal excitation degrees of freedom of the ${ }^{208} \mathrm{~Pb}$ nucleus. 
However, on keeping ${ }^{208} \mathrm{~Pb}$ rigid and removing the rigid-body constraint on ${ }^{24} \mathrm{Mg}$ the cross sections at higher energies are not significantly changed as compared to the previous calculation, but are now enhanced significantly at lower energies. This indicates that not only the reorientation of the deformed nucleus but its internal excitations is also important at lower energies. Relaxing the rigidbody constrains on both the nuclei results in further enhancement of fusion cross sections at lower energies.

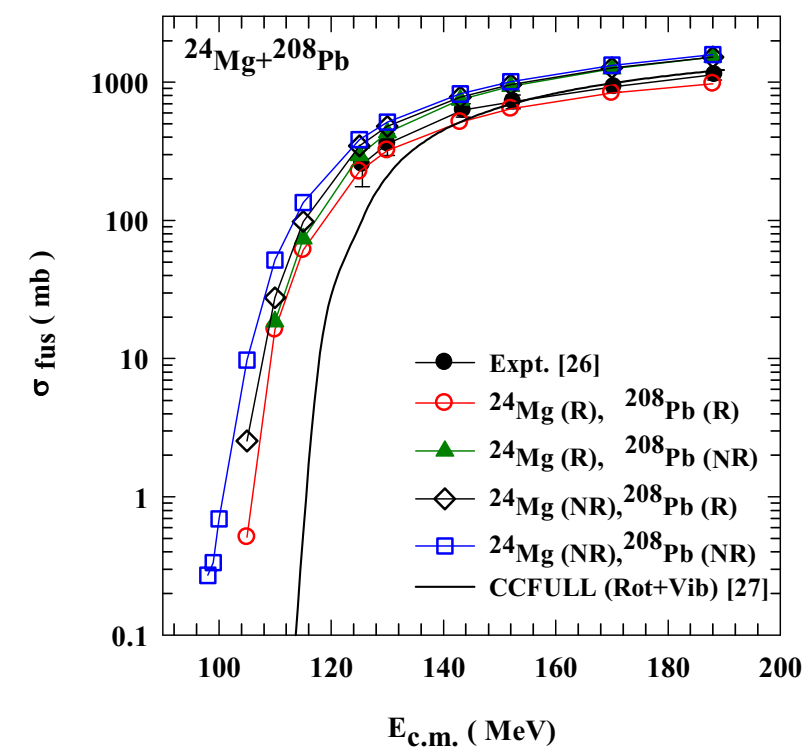

Figure 3: Fusion cross sections for ${ }^{24} \mathrm{Mg}+{ }^{208} \mathrm{~Pb}$ system.

Experimental fusion cross sections [26] are also shown in the figure-3 which are, however, not available below $\mathrm{E}_{\mathrm{cm}}=125.5 \mathrm{MeV}$. Although, the calculations where both the nuclei are kept rigid (CRBD) matches reasonably well with the experimental data in figure-3, however it should be noted that as a result of opening up of internal excitation degrees of freedom the calculated cross sections are enhanced in general. Large enhancement compared to the experimental data in the present calculation is due to the larger radius of the heavy target constructed in this calculation (table-1).

A coupled channel calculation [27] for this reaction using CCFULL code [6] where Wood-Saxon parameters are determined by fitting to the higher energy data and including $2^{+}$rotational coupling for ${ }^{24} \mathrm{Mg}$ and single phonon vibrational $\left(3^{-}\right)$coupling for ${ }^{208} \mathrm{~Pb}$ is also shown in figure-3. However, this CCFULL calculation does not match well with the experimental data at lower energies.

\section{Conclusions}

The 3S-CMD model presented here combines the CRBD and CMD models seamlessly in a single calculation. Doing so is better than using either of the two approaches alone. It combines the benefits of both the approaches as it takes into account both, (i) the reorientation effect which is a long-range effect, and (ii) the internal excitations which occur close to the barrier or inside of it.

Calculated fusion cross sections for ${ }^{24} \mathrm{Mg}+{ }^{208} \mathrm{~Pb}$ show enhancements as the degrees of freedom are increased by relaxation of rigid-body constraints on one or both the colliding partners.

The 3S-CMD approach is easily extended to simulate heavy-ion collisions involving stable weakly-bound projectiles composed of two tightly bound constituent nuclei in a 3-body, 3S-CMD approach [21, 22].

\section{Acknowledgments}

The author would like to thank Mitul R. Morker for help with computing. A part of this work was carried out with the financial assistance under a DAE-BRNS project no. 2009/37/20/BRNS.

\section{References}

1. M. Dasgupta et al, Annu. Rev. Nucl. Part. Sci. 48, 401 (1998); and references therein

2. S. S. Godre, P. R. Desai, Nucl. Phys. A 834, 195c (2010)

3. P. R. Desai, S. S. Godre, Eur. Phys. J. A 47, 146 (2011)

4. J. R. Birkelund et al, Phys. Rep. 56, 107 (1979)

5. A. Diaz-Torres et al, Phys. Rev. Lett. 98, 152701 (2007)

6. K. Hagino, N. Rowley, A. T. Kruppa, Comput. Phys. Commun. 123, 143 (1999)

7. P. Bonche et al, Nucl. Phys. A 443, 39 (1985)

8. C. Simenel, Ph. Chomaz, G. de France. Phys. Rev. Lett. 93, 102701 (2004)

9. A. S. Umar, V. E. Oberacker, Phys. Rev. C 74, 024606 (2006)

10. K. Washiyama, D. Lacroix, Phys. Rev. C 78, 024610 (2008)

11. A. R. Bodmer, C. N. Panos, Phys. Rev. C 15, 1342 (1977)

12. J. J. Molitoris et al, Phys. Rev. Lett. 53, 899 (1984)

13. S. M. Kiselev, Phys. Lett. B 154, 247 (1985)

14. A. Vicentini, G. Jacucci, V. R. Pandharipande, Phys. Rev. C 31, 1783 (1985)

15. V. S. Rammurthy, S. K. Kataria, Pramana-J. Phys. 11, 457 (1978)

16. S. S. Godre, Y. R. Waghmare, Phys. Rev. C 36, 1632 (1987)

17. P. R. Desai, S. S. Godre, Proc. Int. Symp. on Nucl. Phys. 54, 294 (2009)

18. I. B. Desai, S. S. Godre, Proc. Int. Symp. on Nucl. Phys. 54, 196 (2009)

19. S. S. Godre, P. R. Desai, Proc. Symp. on Nucl. Phys. 53, 341 (2008)

20. M. R. Morker, S. S. Godre, Proc. Symp. on Nucl. Phys. 57, 560 (2012)

21. M. R. Morker, S. S. Godre, Proc. Symp. on Nucl. Phys. 56, 644 (2011)

22. M. R. Morker, S. S. Godre, this proceeding (2014)

23. Y. R. Waghmare, Phys. Rev. 136, B1261 (1964)

24. W. D. Myers, W. J. Swiatecki, Ann. Phys. (N.Y.) 55, 395 (1969)

25. C. Y. Wong, Phys. Rev. Lett. 31, 766 (1973)

26. B. B. Back, Phys. Rev. C 31, 2104 (1985)

27. N. Chauhan, S. S. Godre, Proc. Symp. on Nucl. Phys., 56, 640 (2011) 